\title{
Bioinspired and osteopromotive polydopamine nanoparticle-incorporated fibrous membranes for robust bone regeneration
}

\author{
Yi Deng ${ }^{1,2,3}$, Wei-Zhong Yang ${ }^{2}$, Dong Shi ${ }^{1}$, Minjie Wu', Xiao-Ling Xiong ${ }^{1}$, Zhi-Gang Chen $\mathbb{E}^{4,5}$ and Shi-Cheng Wei,
}

\begin{abstract}
Due to their inherent hydrophobic and bioinert nature, synthetic degradable polymer-based membranes show inferior stem cell attachment, proliferation, and even differentiation. To overcome these limitations, bioinspired and osteopromotive polydopamine nanoparticle-incorporated fibrous membranes are developed via a two-step route: $\mathrm{pH}$ induced polymerization of dopamine and co-electrospinning of polycaprolactone $(\mathrm{PCL})$ with polydopamine nanoparticles (PDA NPs). Hybrid membranes with optimized PDA NP content exhibit high quantities of apatite deposition and prominent cytocompatibility (cell attachment, spreading and reproduction) and osteo-differentiation potential (alkaline phosphatase activity, calcium mineralization, and osteogenesis-related genes and protein expression) of human mesenchymal stem cells cultured without any growth factors. Importantly, in vivo assessments using a mouse calvarial critical-sized defect demonstrate that the engineered fibrous membranes remarkably boost bone reconstruction and regeneration. Accordingly, our bioinspired PCL-based hybrid fibrous membranes with robust osteoinductive ability can potentially be utilized as a clinically applicable candidate in guided tissue regeneration applications.
\end{abstract}

\section{Introduction}

Bone defects and injuries (e.g., surgical resections, nonunions, and fractures) are common microscopic defects because of diseases, congenital abnormalities, accidents, and military injuries ${ }^{1-3}$. Most of these cannot be healed spontaneously via a self-repairing mechanism. As a direct result, there is a pressing requirement for developing robust biomaterials that facilitate bone formation by manipulating stem cell lineage commitment into an osteoblast. Guided tissue regeneration (GTR)

\footnotetext{
Correspondence: Wei-Zhong Yang (ywz@scu.edu.cn) or Zhi-Gang Chen (zhigang.chen@usq.edu.au) (zhigang.chen@uq.edu.au)

Shi-Cheng Wei (sc-wei@pku.edu.cn)

${ }^{1}$ Central Laboratory, Peking University School and Hospital of Stomatology,

National Engineering Laboratory for Digital and Material Technology of

Stomatology, Beijing 100081, China

${ }^{2}$ School of Chemical Engineering and College of Materials Science and

Engineering, Sichuan University, Chengdu 610065, China

Full list of author information is available at the end of the article.
}

strategies based on the use of membranes have been highly recommended for large-scale bone regeneration ${ }^{4-6}$. Electrospun membranes have captured tremendous attention in recent years for guided tissue engineering applications considering the following advantages: (1) the membranes can mimic the niche where stem cells reside ${ }^{7}$; (2) the porous fibers possess a large surface area, which provides a friendly environment to support cell attachment and growth; and (3) the 3D fibrous micromilieu of membranes dramatically accelerates the osteogenic potential of numerous stem cell lines, including mouse osteoblastic progenitor cells, rat/human mesenchymal stem cells, human adipose stem cells (hASCs), and human pluripotent stem cells (hPSCs) ${ }^{8-10}$. For instance, a composite nanofibrous membrane consisting of poly(L-lactic acid)/poly(benzyl-L-glutamate)/collagen (PLLA/PBLG/ Col) was prepared through electrospinning, possessing the capability to enhance the osteogenic conversion of

\section{(c) The Author(s) 2019}

(c) (i) Open Access This article is licensed under a Creative Commons Attribution 4.0 International License, which permits use, sharing, adaptation, distribution and reproduction cc) in any medium or format, as long as you give appropriate credit to the original author(s) and the source, provide a link to the Creative Commons license, and indicate if changes were made. The images or other third party material in this article are included in the article's Creative Commons license, unless indicated otherwise in a credit line to the material. If material is not included in the article's Creative Commons license and your intended use is not permitted by statutory regulation or exceeds the permitted use, you will need to obtain permission directly from the copyright holder. To view a copy of this license, visit http://creativecommons.org/licenses/by/4.0/. 
hASCs without an inducing medium ${ }^{8}$. Compared to cell culture plates, peptide-decorated polycaprolactone (PCL) fibers significantly improved the osteo-differentiation efficiency of hPSCs to osteoblasts ${ }^{11}$.

Synthetic polymers applied for GTR membranes are mainly divided into two categories: nonabsorbable and bioabsorbable. A second surgery is needed to eliminate nonabsorbable membranes, which increases healthcare expenditure for patients and compromises the newly generated tissue. Biodegradable membranes composed of polyvinyl alcohol (PVA), poly(lactic acid) (PLA), poly (lactide-co-glycolide) (PLGA) or PCL are preferred in clinical implantation ${ }^{12,13}$. However, these existing bioabsorbable polymers have some severe concerns, including high hydrophobicity and lack of cell affinity moieties, so they are not beneficial for stem cell attachment/growth and tissue integration, and their clinical adoption is seriously impeded. PCL, a USA Food and Drug Administration authorized biomaterial, has aroused much interest due to its low melting point $\left(56-61{ }^{\circ} \mathrm{C}\right)$, slow degradation, acceptable cytocompatibility, and excellent blend compatibility $^{14}$. Inspired by the adhesive proteins secreted from mussels, a material-independent and facile surface coating engineering strategy based on catechol chemistry has gained prominence since Messersmith and colleagues discovered the $\mathrm{pH}$-triggered self-polymerization of dopamine ${ }^{15}$. Previous literature has demonstrated that polydopamine (PDA) possesses a strong capability to promote cell adhesion ${ }^{16}$ and facilitates the osteoblastic differentiation of stem cells on various substrates ${ }^{17,18}$. The PDA nanoscale coating on biomaterials has been found to not only support mesenchymal stem cell attachment ${ }^{19}$ but also induce reprogramming of human somatic cells and maintain long-term self-renewal of hPSCs under chemically defined conditions ${ }^{20}$. However, the PDA nanolayers easily detached from surfaces when these coated biomaterials suffered from physical stimulation or remained in the body for a long time. Unfortunately, the detached PDA debris will certainly induce local cell apoptosis ${ }^{21}$ and inflammatory reactions ${ }^{22}$, which are detrimental to tissue regeneration. The blending of PDA into the polymer matrix may be an excellent option to reduce the detachment of PDA, ultimately eliminating its adverse effect on cells and tissues. Despite the rapid advances in the development of catechol-based surface engineering (i.e., PDA-coated biomaterial surfaces), the use of PDA nanoparticles (PDA NPs) as a biofunctional additive in electrospun fibrous membranes for guided tissue regeneration applications has not been reported, to the best of our knowledge. Herein, the goals of this work are to (1) fabricate PDA-incorporated PCL (PDA/PCL) hybrid fibrous membranes by electrospinning dispersed PDA NPs within a synthetic polymer carrier; (2) assess the chemical and physical properties of the microfibrous membranes; (3) investigate how hMSCs in vitro respond to the engineered scaffolds regarding adhesion, proliferation and osteogenic differentiation; and (4) evaluate the in vivo osteogenesis ability over 2 months in a mouse calvarial critical size defect model. Our developed fibrous membranes provide a favorable niche to steer the fates of local stem cells toward osteoblasts for bone regenerative medicine.

\section{Experimental section \\ Preparation of PDA NP-incorporated membranes}

First, $2 \mathrm{mg} / \mathrm{mL}$ of a dopamine solution (Sigma, USA) was fabricated by dissolving it in $10 \mathrm{mM}$ of a Tris- $\mathrm{HCl}$ solution $(\mathrm{pH}=8.5)$ with stirring for 1 day at $60^{\circ} \mathrm{C}$. Then, the reaction was stopped by centrifugation at $5000 \mathrm{rpm}$, followed by drying at $60^{\circ} \mathrm{C}$ to obtain PDA NP powders.

PCL granules $(\mathrm{Mw} \approx 80,000 \mathrm{~g} / \mathrm{mol}$, Sigma-Aldrich) with different amounts of PDA NPs (mass ratio of PCL:PDA NPs $=100: 1,100: 2,100: 5$, and 100:10) were dissolved in hexafluoroisopropanol (HFIP) through ultrasonic agitation overnight to achieve a concentration of $8 \%(\mathrm{w} / \mathrm{v})$. After that, the prepolymer solution was transferred to a syringe with a $23 \mathrm{G}$ blunt needle. The electrospinning of fibrous membranes was conducted by a conventional electrospinning setup (SS-2535, YongKang Technology, China) according to our previous work ${ }^{23}$. The resulting membranes were collected on $\mathrm{Al}$ films and then vacuumdried to remove the residual organic solvent. According to the different concentrations of PDA NPs $(1,2,5$, and $10 \mathrm{wt} \%$ ), these membranes were denoted as $1 \% \mathrm{PDA} / \mathrm{PCL}$, 2\% PDA/PCL, 5\% PDA/PCL, and 10\% PDA/PCL.

\section{Material characterization}

Microstructural analysis of fibrous membranes was investigated with a scanning electron microscope (SEM, JSM-6701F, JEOL, Japan). The chemical analysis of the engineered fibrous membranes was identified through Raman spectroscopy and X-ray photoelectron spectroscopy (XPS, Kratos Analytical, UK). The hydrophilicity of the membranes was tested through contact angle goniometry (SL200B, Kino, USA).

\section{Formation of bone-like apatite}

The $1 \times$ synthetic body fluid (SBF) (Table S1) was fabricated by dissolving $\mathrm{NaCl}, \mathrm{NaHCO}_{3}, \mathrm{KCl}$, $\mathrm{K}_{2} \mathrm{HPO}_{4} \cdot 3 \mathrm{H}_{2} \mathrm{O}, \mathrm{MgCl}_{2} \cdot 6 \mathrm{H}_{2} \mathrm{O}, \mathrm{CaCl}_{2}$, and $\mathrm{Na}_{2} \mathrm{SO}_{4}$ in order in deionized water and buffering to $\mathrm{pH} 7.4$ using $\left(\mathrm{CH}_{2} \mathrm{OH}\right)_{3} \mathrm{CNH}_{2}$ and $1 \mathrm{~mol} / \mathrm{L} \mathrm{HCl}$ at $37^{\circ} \mathrm{C}$. The engineered fibrous membranes were soaked in SBF at $37^{\circ} \mathrm{C}$ to examine the in vitro bioactivity. After 7 and 14 days, samples were taken away from SBF and gently washed. The chemical constitution and microstructure of nodules were determined by XPS and SEM. 


\section{hMSC culture and seeding}

Circular samples $\left(1.9 \mathrm{~cm}^{2}\right)$ were cut from the fibrous membranes and placed into 24-well plates. Afterwards, the samples were disinfected using $75 \%$ ethanol for $40 \mathrm{~min}$ followed by UV light for $30 \mathrm{~min}$ and were then washed using sterile PBS before cell culture. The hMSCs were purchased from Sciencell Research Laboratories (USA) and were extracted from healthy human bone marrow. They were expanded in standard growth medium consisting of highglucose DMEM (HyClone, USA), complemented with 10\% fetal bovine serum (HyClone, China), and $1 \%$ penicillin-streptomycin (P-S) and were then incubated under a $5 \% \mathrm{CO}_{2}$ atmosphere. Cells before passage five were used, and the trypsinized cells were seeded on the microfibers at $2 \times 10^{4}$ cells $/ \mathrm{cm}^{2}$. After 5 days of incubation, the medium was changed to an osteoinductive solution composed of low-glucose DMEM containing 10\% FBS, 1\% P-S, $50 \mu \mathrm{g} / \mathrm{mL}$ ascorbic acid, $10 \mathrm{mM}$ sodium $\beta$-glycerophosphate, and $100 \mathrm{nM}$ dexamethasone (Sigma). The medium was refreshed every $2-3$ days.

\section{Cytocompatibility of membranes Early attachment assay}

The early cellular attachment on the engineered fibrous membranes was conducted using SEM, F-actin fluorescence staining, and the Cell Counting Kit-8 assay (CCK-8, Dojindo, Japan) after incubating for 6 and $12 \mathrm{~h}$. The morphologies of hMSCs on the fibrous membranes were observed using SEM. The substrates with cells were fixed in $2.5 \%$ glutaraldehyde for $30 \mathrm{~min}$ and then dehydrated with graded concentrations of ethanol. Finally, the dehydrated samples were dried in a vacuum dryer and visualized under SEM. The rinsed hMSCs were fixed with $4 \%$ paraformaldehyde for $0.5 \mathrm{~h}$ and permeabilized with $0.1 \%$ Triton X-100. Then, $5 \mu \mathrm{g} / \mathrm{mL}$ of FITC-phalloidin (Sigma) was added to stain the hMSCs for $0.5 \mathrm{~h}$. After rinsing with PBS, the samples were reacted for 8 min with $15 \mu \mathrm{g} / \mathrm{mL}$ DAPI (Sigma). Finally, the fluorescence signal was captured under a confocal laser scanning microscope (CLSM). At the desired time, CCK-8 was introduced into each well at a $10 \%$ proportion for $3 \mathrm{~h}$ in the dark. The optical density (O.D.) of the supernatant was measured by a microplate reader (Model 680, Bio-Rad, USA) according to the manufacturer's instructions.

\section{Cell proliferation assay}

The CCK- 8 kit was also used to measure cell proliferation for 6 days, and the cellular morphologies on the fibrous surfaces at 3 days were observed using SEM.

\section{Osteogenic potential evaluation \\ Alkaline phosphatase activity and mineralization}

The intracellular alkaline phosphatase activity of the hMSCs was evaluated with the alkaline phosphatase
(ALP) assay kit (Nanjing Jiancheng Bioengineering Institute, China) following the manufacturer's specification. The results were normalized to the total protein amount obtained from the MicroBCA protein assay kit (Thermo, USA). Furthermore, the ALP distribution on fibrous membranes was viewed using a BCIP/NBT ALP color development kit (Beijing ComWin Biotech, China).

Mineralized matrix formation was examined after 14 and 21 days of osteoinductive culture by staining with Alizarin Red S (ARS, Sigma). The cell layers were fixed and soaked in the ARS solution $(2 \mathrm{w} / \mathrm{v} \%, \mathrm{pH}=4.2)$ for $0.5 \mathrm{~h}$. After rinsing using deionized water, the images were captured by a scanner. To further quantify mineralization, the ARS-stained substrates were immersed in a hexadecylpyridinium chloride solution $(100 \mathrm{mM}$, Sigma) for $12 \mathrm{~h}$ to dissolve the calcified matrix. The O.D. of the solution was measured at $570 \mathrm{~nm}$ using a microplate reader.

\section{Real-time PCR}

At 2 and 3 weeks of osteoinduction, the total mRNA was isolated from the cells by TRIzol (Invitrogen, USA) treatment and converted into cDNA using the RevertAid First Strand cDNA Synthesis Kit (Thermo, USA) according to the manufacturer's instructions. RT-PCR analysis was performed on an ABI 7500 real-time PCR machine (Applied Biosystems, USA) using SYBR Green (Roche, USA). Each specimen was run in triplicate, and glyceraldehyde 3-phosphate dehydrogenase (GAPDH) was assigned as an endogenous control. The primers $\left(5^{\prime}\right.$ $3^{\prime}$ ) used in this study are listed in Table S2.

\section{Immunofluorescence staining}

After fixation, permeabilization, and treatment with a $1 \%$ BSA/PBST solution, the hMSCs were then exposed to primary antibodies against ALP (1:400, CST), RunX-2 (1:400, CST), OCN (1:400, CST), and Col1a1 (1:400, CST) at $4{ }^{\circ} \mathrm{C}$ for $24 \mathrm{~h}$, rinsed with PBS three times, and incubated with FITC-488 goat anti-rabbit (1:100, ZSGB-BIO, Beijing, China) or TRITC-543 goat anti-mouse (1:100, ZSGB-BIO) secondary antibodies for $60 \mathrm{~min}$. The cells were counterstained with DAPI $(10 \mu \mathrm{g} / \mathrm{mL})$ for nucleus staining and imaged by CLSM.

\section{In vivo bone formation tests Mouse skull defect model}

The animal experiment was approved by the Institutional Animal Care and Use Committee at Peking University (approval no.: AAIS-WeiSC-3). The effect of fibrous membranes on in vivo bone regeneration was investigated using 6- to 8-week-old CD-1 mice. Before the operation, the mice were anesthetized by pentobarbital sodium $(3 \%, 40 \mathrm{mg} / \mathrm{kg})$ through intraperitoneal injection. Two critical-sized defects $(4 \mathrm{~mm}$ diameter) were created 


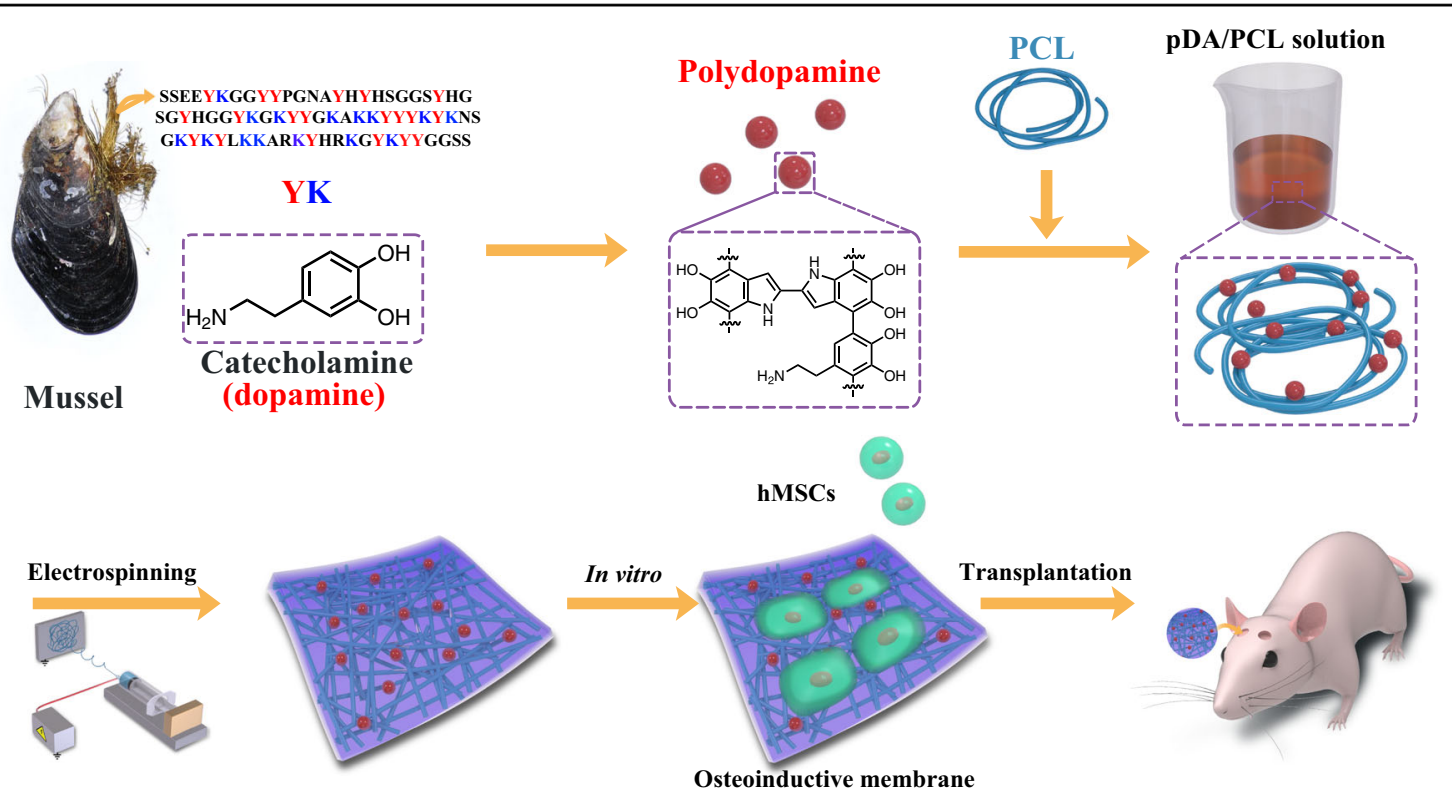

Fig. 1 Schematic drawing of the fabrication of the engineered membranes via electrospinning of PCL and PDA NPs

on both sides of the skull using a dental trephine ${ }^{24}$. The right-side cavity was covered with microfibers, while the left one remained without any fiber implantation and served as the negative control. The animals were divided into three groups based on the implanted membranes: Defect only $(n=6)$, pure PCL microfibers $(n=6)$, and $2 \%$ PDA/PCL fibrous membranes $(n=6)$. To label the mineralization process, the mice were injected intraperitoneally with calcein $(50 \mathrm{mg} / \mathrm{kg}$, Sigma-Aldrich) and ARS $(50 \mathrm{mg} / \mathrm{kg})$ at 1 and 2 weeks, respectively. These mice were euthanized at a scheduled time. After sacrifice, the calvaria was harvested and fixed in $10 \%$ formalin solution for further evaluation.

\section{Micro-CT analysis}

The samples were measured by micro-CT scanning (18 $\mu \mathrm{m}$ voxel, Scanco Medical vivaCT40, Switzerland) to reconstruct 3D images. Afterwards, the trabecular bone volume/tissue volume (BT/TV), trabecular number ( $\mathrm{Tb}$. $\mathrm{N})$, trabecular thickness ( $\mathrm{Tb}$. Th), and trabecular spacing (Tb. Sp) were calculated using the built-in program.

\section{Histological analysis}

To obtain decalcified paraffin sections, the fixed samples were exposed to a 15\% EDTA solution for 3 weeks. After dehydration, the samples were embedded in paraffin and cut into $10 \mu \mathrm{m}$ longitudinal sections for H\&E, Masson trichrome, toluidine blue and immunohistochemical OCN staining via an optical microscope (TE200-E, Nikon Eclipse, Japan). The fluorochrome-labeled samples were dehydrated using different concentrations of ethanol and embedded in methyl methacrylate. Moreover, transverse sections $(10 \mu \mathrm{m})$ were cut to observe the fluorochrome labels under CLSM.

\section{Statistical analysis}

For quantitative experiments, six samples of each group were tested to provide the mean and standard deviation. One-way ANOVA and Tukey's post hoc tests were applied to determine the significant differences among the groups.

\section{Results and discussion \\ Physicochemical properties of PDA/PCL fibrous membranes}

Figure 1 illustrates the fabrication process of the PDA/ PCL fibrous membranes. First, pDA NPs are synthesized via catechol chemistry, and the suspension was centrifuged to obtain pDA NP powders. The microstructure of PDA NPs is shown in Fig. 2a, and spherical particles with an average size of $180-220 \mathrm{~nm}$ are found from the particle size analysis (Fig. S1a) and TEM data. In weakly alkaline conditions $(\mathrm{pH}=8.5)$, dopamine molecules selfpolymerize to form homogeneous pDA NPs. Next, the resulting pDA NPs are uniformly dispersed in the PCL solution through continuous sonication and vortexing to form hybrid fibrous membranes through electrospinning. The surface morphology and diameter of PDA/PCL fibrous membranes with different PDA contents are observed by SEM (Fig. 2b). The membranes are made of a randomly micrometer-sized fibrous network. Pure PCL electrospun membranes display smooth surfaces with a 

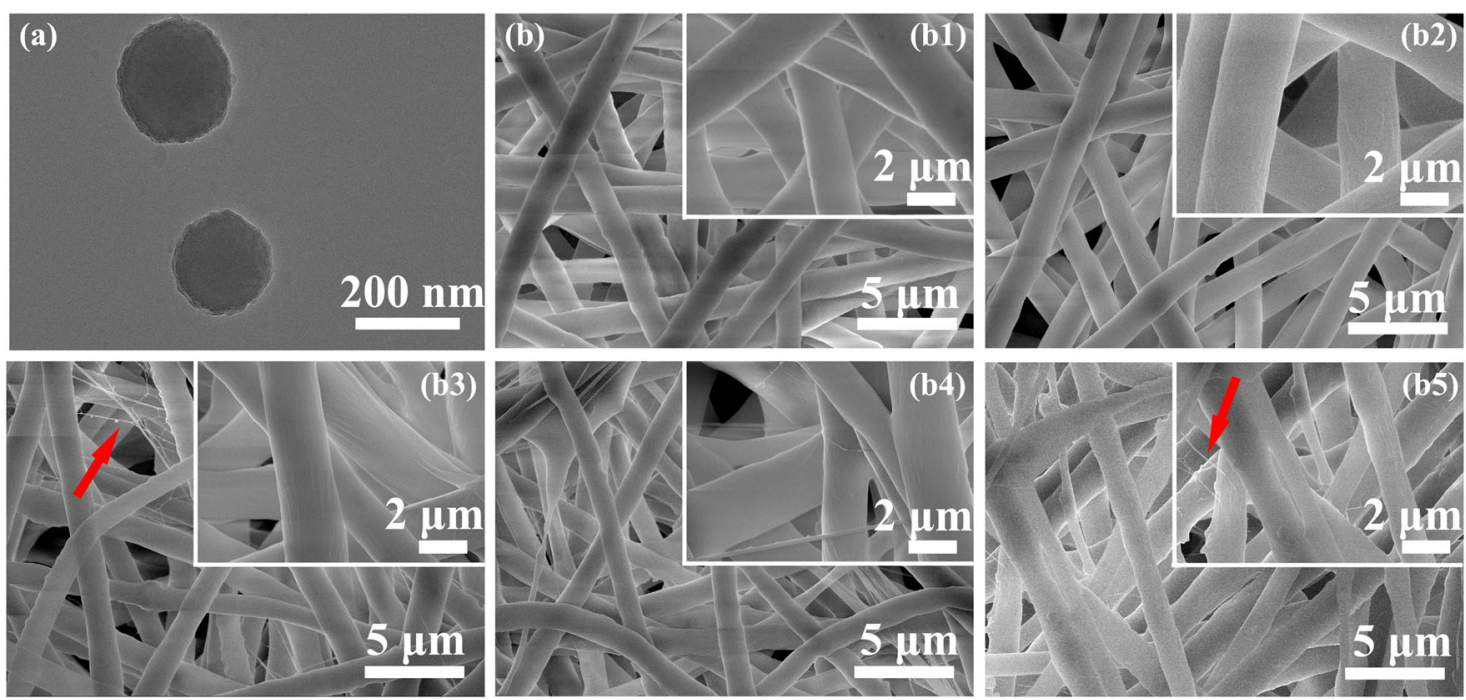

Fig. 2 Micromorphologies of PDA NPs and electrospun PDA/PCL fibrous membranes. a TEM image of PDA NPs; $\mathbf{b}$ SEM images of the (b1) pristine PCL, (b2) 1\% PDA/PCL, (b3) 2\% PDA/PCL, (b4) 5\% PDA/PCL, and (b5) 10\% PDA/PCL fibrous membranes. The red arrows in $\mathbf{b}$ point to the filaments

(a)

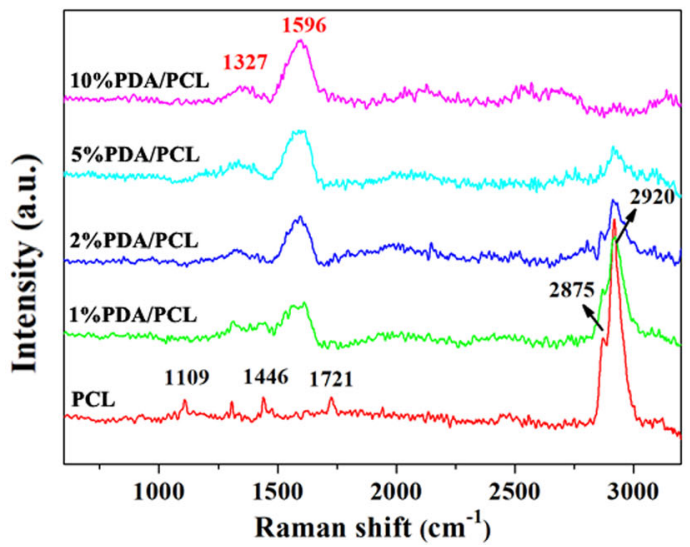

(b)

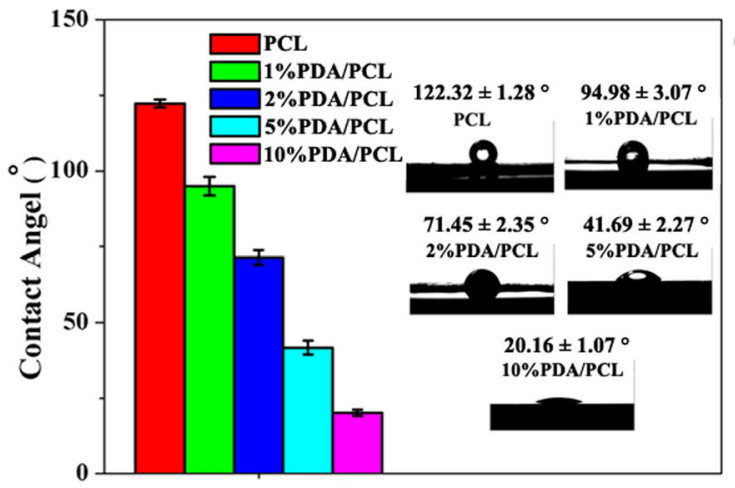

Deionized water

Fig. 3 Chemical constituents of PDA/PCL fibrous membranes. a Raman spectra and $\mathbf{b}$ contact angles with corresponding water droplet images of PDA/PCL fibrous membranes

diameter of $1.47 \pm 0.04 \mu \mathrm{m}$. After the incorporation of PDA NPs, the fiber surfaces were roughened, and some filaments were observed under a high amount of PDA NPs. In addition, ImageJ analysis shows that the diameter of the fibrous membranes remarkably decreases with increasing PDA NP content.

Raman spectra were collected to identify the existence of PDA NPs. Figure 3a shows that pure PCL fibrous membranes present characteristic peaks of $\mathrm{C}-\mathrm{H}$ stretching vibration at 2875 and $2920 \mathrm{~cm}^{-1}, v(\mathrm{C}=\mathrm{O})$ stretching vibration at $1721 \mathrm{~cm}^{-1}, \omega(\mathrm{CH} 2)$ vibration at $1446 \mathrm{~cm}^{-1}$, and $\mathrm{C}-\mathrm{O}-\mathrm{C}$ symmetric stretching vibration at $1109 \mathrm{~cm}^{-1}$. For PDA/PCL fibrous membranes, the apparent bands at
1596 and $1327 \mathrm{~cm}^{-1}$ are associated with the deformation and stretching, respectively, of catechol from $\mathrm{PDA}^{25}$. Moreover, the intensity of catechol peaks increases along with a reduction of PCL peaks with increasing PDA contents in the fibrous membranes. These findings are further confirmed by XPS analysis, which can provide direct evidence of the chemical composition. In the full spectrum of pure PCL fibrous membranes (Fig. S2), C and $\mathrm{O}$ are the primary elements. The appearance of an N1s peak in the full spectrum of PDA/PCL fibrous membranes and its enhanced intensity with increasing PDA concentration indicate the successful incorporation of PDA NPs. Additionally, the hydrophilicity of biomaterials has 


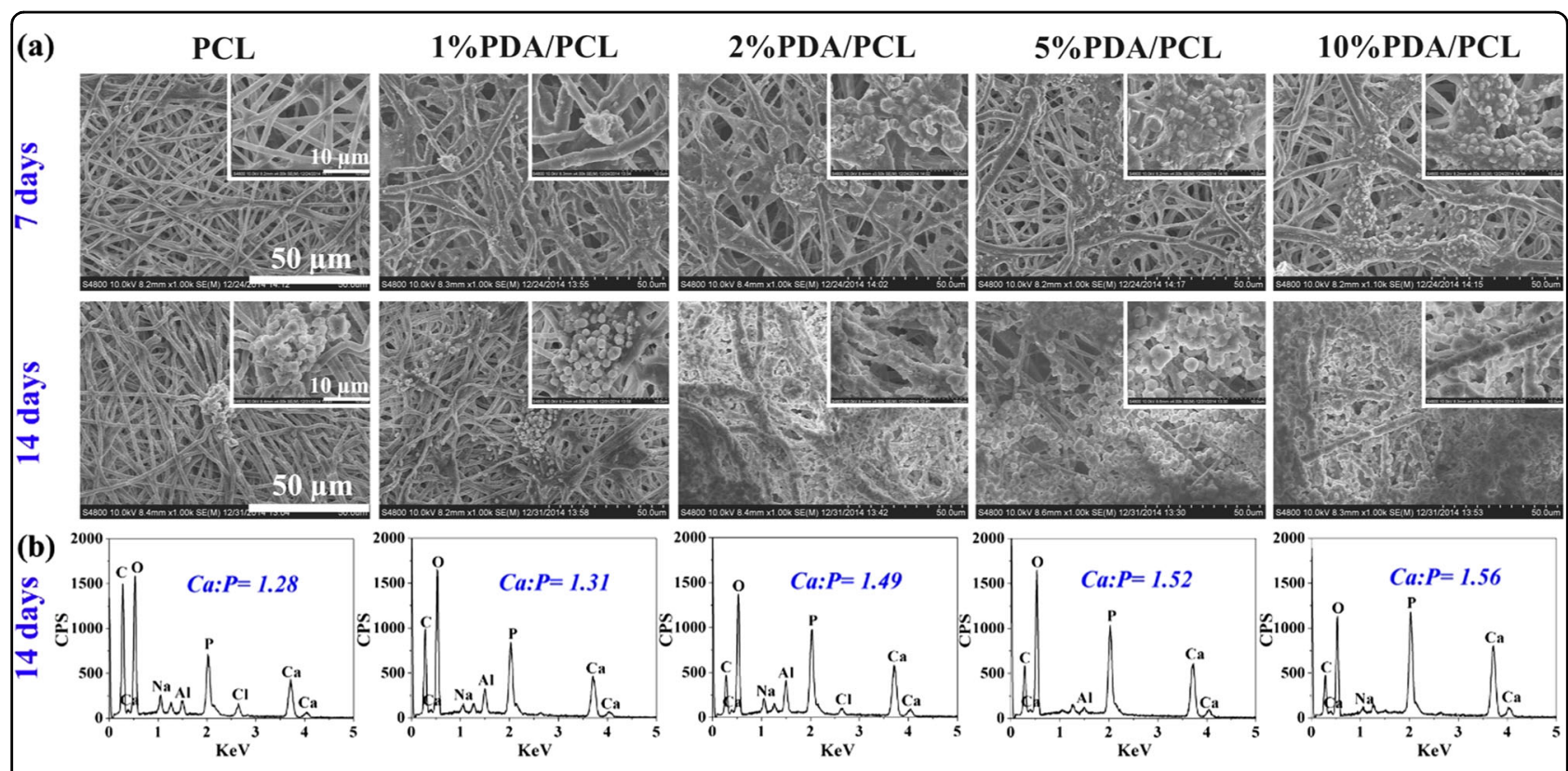

Fig. 4 Bone-like nodules formation. a SEM photographs of PDA/PCL fibrous membranes acquired after soaking in SBF for 7 and 14 days. b EDS data and $\mathrm{Ca} / \mathrm{P}$ ratios of the particle sediments on the surface of PDA/PCL fibrous membranes at 14 days

been well proven to play a crucial role in protein adsorption and cell attachment ${ }^{26}$. The contact angle measurement in Fig. $3 \mathrm{~b}$ suggests that unmodified PCL fibrous membranes are superhydrophobic (122.32 \pm $\left.1.28^{\circ}\right)$. After PDA modification, the hydrophilicity of the membranes dramatically improves, with the 10\% PDA/ PCL samples being the most wettable with a value of $20.16 \pm 1.07^{\circ}$.

\section{Bone-like apatite formation}

One critical feature of bioactive membranes is their capability to integrate with the surrounding osseous tissue; therefore, the formation of bone-like apatite layers on the surface of the materials in SBF solution was evaluated. As shown in Fig. 4, no Ca-P deposition was observed on pristine PCL membranes at the initial 7 days, but several round apatite islands appeared after 14 days of soaking. In contrast, a large quantity of clustered nodular aggregations emerged on the PDA/PCL fibrous membrane surfaces after 7 days of immersion, while with the extension of time to 14 days, the amount of deposition on PDA/PCL fibrous membranes dramatically increased. Interestingly, when the amount of PDA is $>2 \%$, apatite covers the entire surface of the fibrous membranes, forming an apatitedecorated fiber structure. We can see that the amount of deposited apatite increases with increasing PDA content. EDS detection of the nodules reveals that the depositions contain $\mathrm{Ca}$ and $\mathrm{P}$ with ratios in the range of 1.28-1.56, which is lower than the $\mathrm{Ca} / \mathrm{P}$ stoichiometric ratio $(=1.67)$ in hydroxyapatite. However, a higher $\mathrm{Ca} / \mathrm{P}$ ratio was detected in the fibrous membranes with a higher content of PDA NPs, indicating that PDA contributes to the in vitro formation of apatite that is much closer to HA. XPS was further used to detect the chemical constituents of deposited particles on the 2\% PDA/PCL fibrous membranes immersed in SBF for 7 and 14 days. Figure S3 shows that the Ca 2p signal exhibits a doublet at 347.1 and $349.6 \mathrm{eV}$, and the $\mathrm{P} 2 \mathrm{p}$ signal shows a single peak at $132.9 \mathrm{eV}$. The two spectra significantly increased with soaking time, in line with the published data for bone-like apatite. The mechanism of apatite formation on the PDAcontaining fibrous membranes can be explained by the chelation interactions of PDA with ions in the SBF. The PDA phase in the fibrous membranes has numerous catechol moieties on the surface, which trigger heterogeneous nucleation and growth of apatite via the chelation of $\mathrm{Ca}^{2+}$. Positively charged $\mathrm{Ca}^{2+}$ in the SBF is first absorbed to catechol-rich surfaces. Subsequently, anions such as $\mathrm{HPO}_{4}{ }^{2-}$ and $\mathrm{OH}^{-}$in the SBF solution are attracted, contributing to the formation of bone-like apatite crystals. These data show that the in vitro bioactivity of PDA/PCL fibrous membranes is better than that of the pure PCL control.

\section{Cytocompatibility of PDA/PCL fibrous membranes}

We further evaluated the effect of the engineered PDA/ PCL membranes on the cell adhesion, spreading, and proliferation of hMSCs, which exist ubiquitously in bone marrow and have an ability to repair tissue when it suffers injuries. Figure $5 \mathrm{a}, \mathrm{b}$ displays an overview of the 


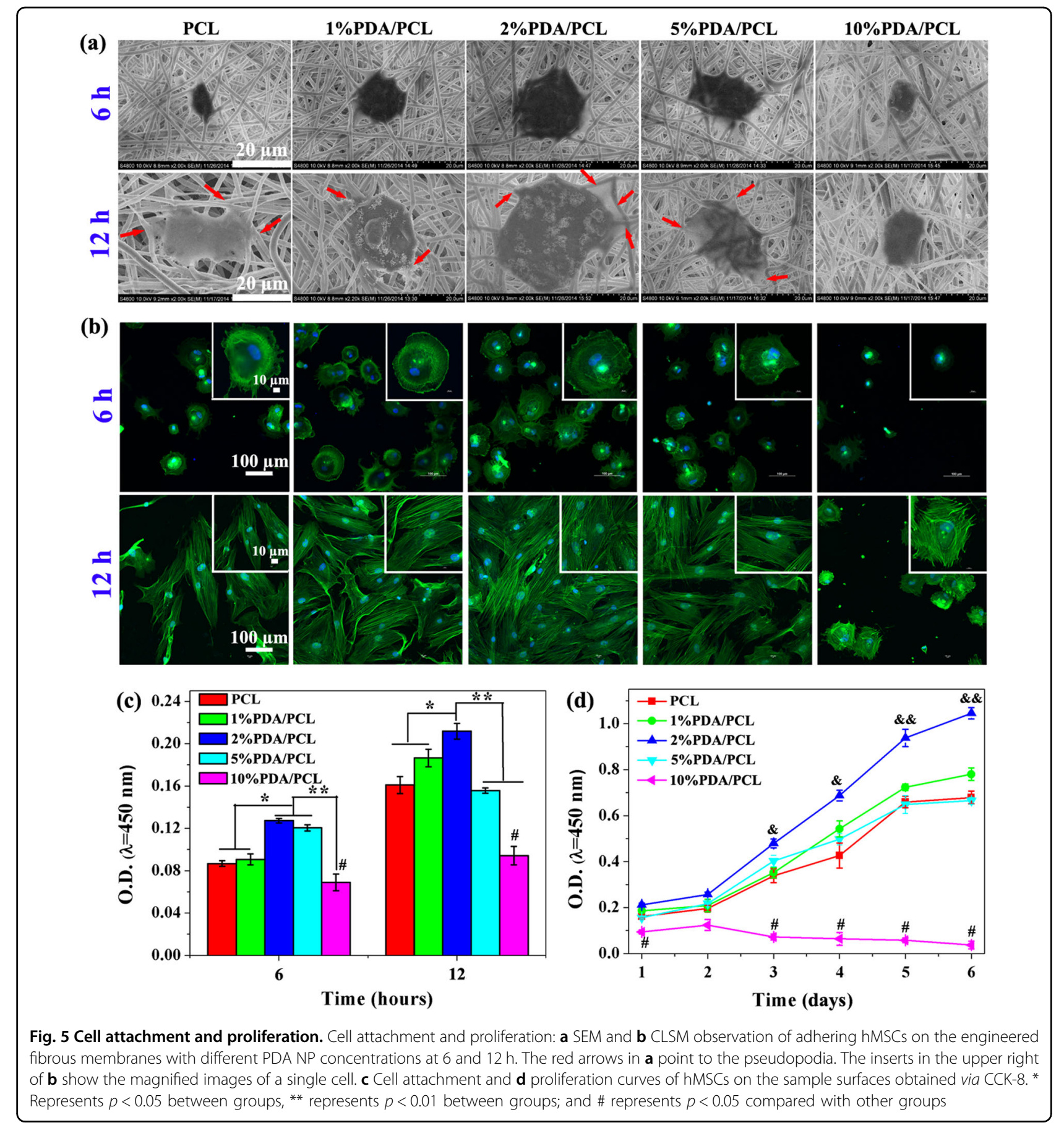

morphology of hMSCs on fibrous membranes captured by SEM and CLSM. Apparently, after $6 \mathrm{~h}$ of culturing, hMSCs with round cellular shapes do not show pseudopodia and express poor F-actin on pure PCL and 10\% PDA/PCL fibrous membranes, but filopodia emerge on the other three PDA/PCL membrane surfaces. At $12 \mathrm{~h}$, Except the cells in the 10\% PDA/PCL group, the attached hMSCs in most of the groups spread with mature F-actin intracellular nanofibers, an indication of the inhibitory effect on cell adhesion. Notably, cells in the 2\% PDA/PCL group show a larger spreading area and more extended filopodia than those in the 1 and 5\% PDA/PCL groups. Furthermore, the cell-counting experiments revealed that the content of PDA NPs in the fibrous membranes has a significant impact on the number of viable cells (Fig. 5c). The most considerable quantity of cells was detected in the $2 \%$ PDA/PCL group, and the fewest cells were found on the $10 \%$ PDA/PCL substrates. 
The CCK- 8 assay was conducted to investigate hMSC proliferation on fibrous membranes. Figure $5 \mathrm{~d}$ shows that the cell viability results in most material groups present excellent time-dependent performance. However, PCL with 10\% PDA NPs dramatically dampens cell growth during the whole incubation period. Compared with that of the other microfibrous substrates, cell viability in the $2 \%$ PDA/PCL group was high after 6 days of incubation, in accordance with the SEM observations (Fig. S4). These results demonstrate that the incorporation of PDA NPs into bioinert PCL fibrous membranes with proper amounts can equip fibrous membranes with acceptable cytocompatibility.

\section{Osteogenic differentiation of hMSCs}

To determine the optimal formulation of the engineered PDA/PCL membranes for guided bone tissue regeneration, the ALP activity and calcium matrix production of hMSCs were measured using ARS staining. Because a high concentration of PDA NPs is toxic to stem cells, the 10\% PDA/ PCL fibrous membranes are not included for the osteogenic differentiation assays. As illustrated in Fig. 6a, b, all fibrous membranes exhibit good time-dependent ALP expression. The $2 \%$ PDA/PCL fibrous membranes possess higher ALP activity than that of the pure PCL and 5\% PDA/PCL groups, although there is no discernible difference in ALP expression between the 1 and 2\% PDA/PCL samples at 14 days. However, a reduction in ALP production was detected for the fibrous membranes with an addition of PDA NPs of up to $5 \%$, indicating that the high concentration of PDA has an adverse effect on hMSC osteo-differentiation. These results are further confirmed through BCIP/NBT ALP staining, where the ALP-positive areas on the $2 \% \mathrm{PDA} / \mathrm{PCL}$ fibrous membranes are larger than that on the other engineered membranes at 7 and 14 days.

Figure 6c, d displays that after 2 and 3 weeks of osteoinductive culture, the amount of PDA NPs in the fibrous membranes influences hMSC mineralization. Considering the possible ARS adsorption on fibrous membranes, the quantitative data are calibrated by excluding the OD value of background staining of the fibrous membranes. In accordance with the ALP results, compared with the pure PCL counterparts, the hybrid fibrous membranes show bright red and positive areas due to the introduction of PDA NPs. In addition, an apparent increase in the formation of calcium nodules was found in the 2\% PDA/PCL fibrous membranes at both 14 and 21 days. The quantitative mineralized production data confirm the ARS staining results. Clearly, positive concentration-dependent cell responses can be observed on the PDA-incorporated fibrous membranes, consistent with previous reports that PDA can accelerate the calcified matrix of hMSCs in a dose-dependent manner ${ }^{17,27}$. Cell proliferation and osteogenic differentiation are low when the amounts of PDA NPs are either high and low because low concentrations of PDA NPs cannot trigger the growth and differentiation of hMSCs, while high concentrations dampen cell reproduction and cause cell death, ultimately leading to inferior osteogenic differentiation, as discussed in the CCK- 8 results.

Investigation at the molecular level is a powerful tool to better understand the interplay between hMSCs and engineered fibrous membranes. Thus, we further monitored the expression of osteogenesis-related genes encoding ALP, RunX2, Col1a1, and OPN in hMSCs cultured on fibrous membranes. ALP is a well-known phosphatase enzyme that is widely employed to show early osteoblastic phenotypic expression during osteogenic differentiation ${ }^{28}$. RunX-2 is a vital transcription factor that regulates numerous other genes related to osteogenesis ${ }^{29,30}$. Collagen (Colla1) and osteopontin (OPN) are bone extracellular matrix proteins and have critical functions during bone regeneration/ remodeling ${ }^{31,32}$. As shown in Fig. 6e, the most substantial amount of ALP gene expression is found in the 2\% PDA/ PCL sample at 7 and 14 days. At 14 days, the level of RunX2 gene expression in the $1 \% \mathrm{PDA} / \mathrm{PCL}$ and $2 \% \mathrm{PDA} / \mathrm{PCL}$ groups significantly outmatches that in the pure PCL group, although there is no discernible difference among the four groups after 21 days of stimulation. Furthermore, the Colla1 level of the 2\% PDA/PCL groups is elevated compared with that of the other fibrous membrane groups at 14 days. OPN expression correlates with changes in Colla1 expression. In particular, the most substantial amount of OPN gene expression is detected in the $2 \%$ PDA/PCL fibrous membranes. The high levels of the four osteogenesis-related genes indicate that osteogenic differentiation is active on the surface of the $2 \%$ PDA/PCL fibrous membranes. The osteoblastic differentiation efficiency of hMSCs on various PDA/PCL fibrous membranes was further verified by the expression of the osteo-related proteins through immunofluorescence staining. Fig. S5 shows that cells on the 1\% PDA/PCL fibrous membranes are characterized by the enhanced expression of osteogenesis-associated proteins, in line with the real-time PCR results. In addition, the expression levels of RunX2, ALP, Collal and OCN (an essential marker in mature osteoblasts) in hMSCs are greatly enhanced on the $2 \%$ PDA/PCL substrates compared to those on the 1\% PDA/ PCL substrates. Therefore, the results from the in vitro osteogenic differentiation assays show that PDA/PCL fibrous membranes with a proper PDA amount (2\%) possess a robust osteoinductive capacity of inducing hMSCs to differentiate into mature osteoblasts.

\section{In vivo bone regeneration assessment}

The in vitro cell experiments data guided us to assess the in vivo biofunctionality of the microfibrous membrane using an animal model. We created critical-sized bone 


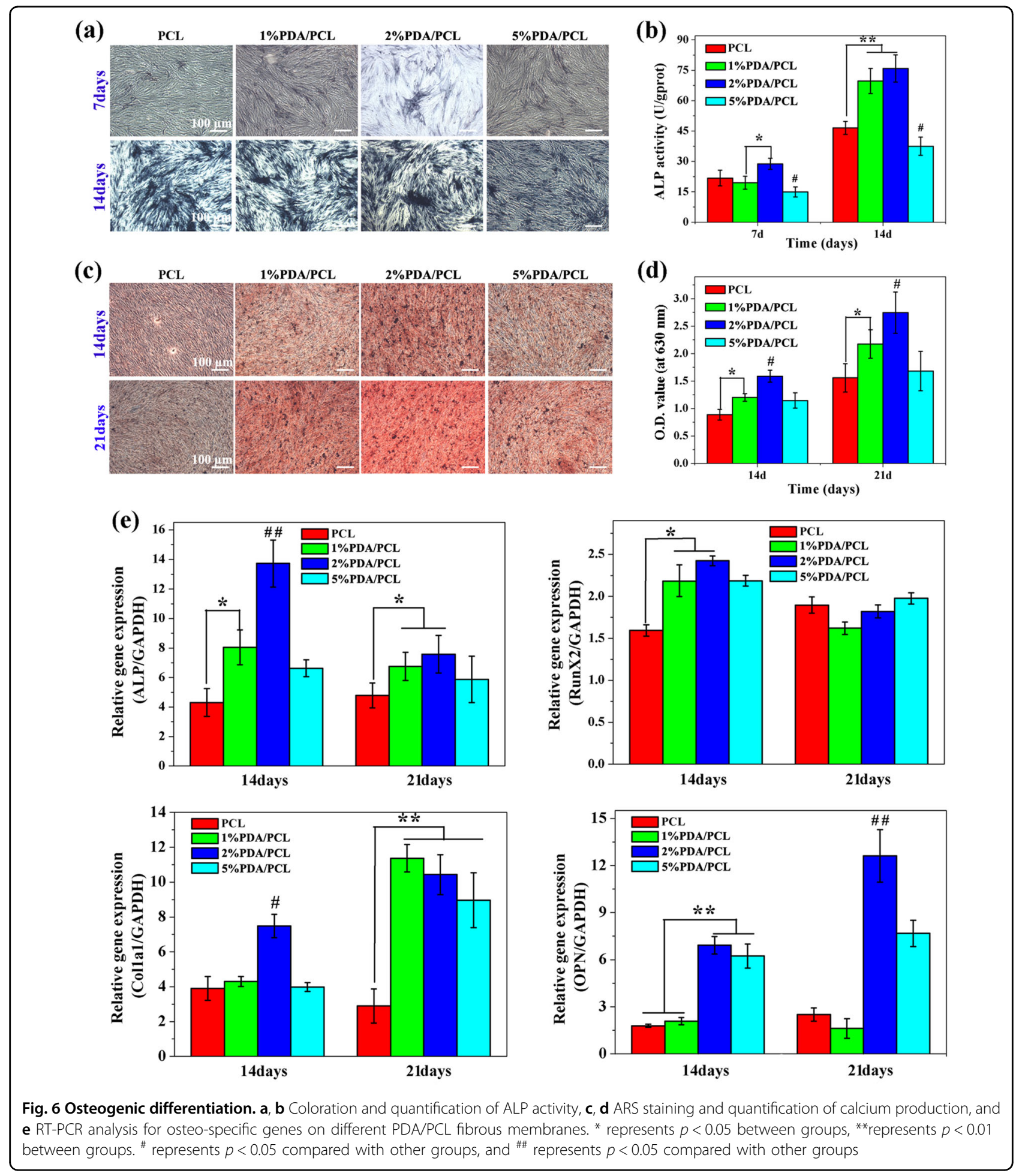

defects on mouse skulls, placed fibrous membranes to cover the defects, and evaluated bone formation through micro-CT, histological analyses and fluorescent labeling at 4 and 8 weeks after transplantation. Representative 3D images reconstructed from the high-resolution micro-CT of skulls containing defects and their quantified bone areas are shown in Fig. 7a-c. At 8 weeks post surgery, limited bone formation is found in the defect-only groups $(16.3 \pm 1.84 \%)$, while a slight improvement is observed in the groups implanted with pure PCL fibrous membranes, showing an enhanced guidance effect. The $2 \% \mathrm{PDA} / \mathrm{PCL}$ membranes show the largest areas occupied by new bone 


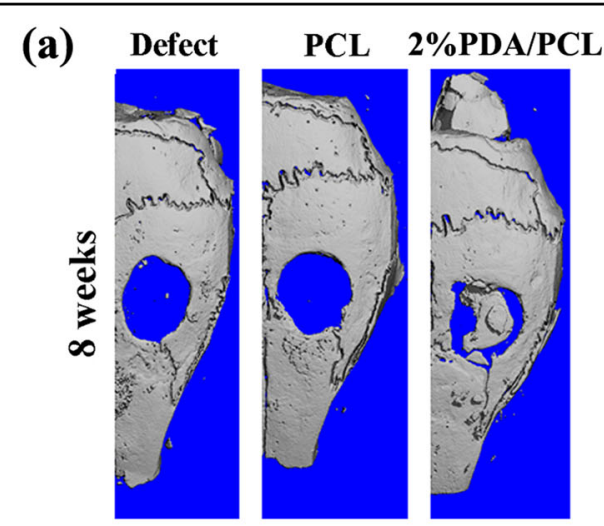

(b)

(c)

Defect
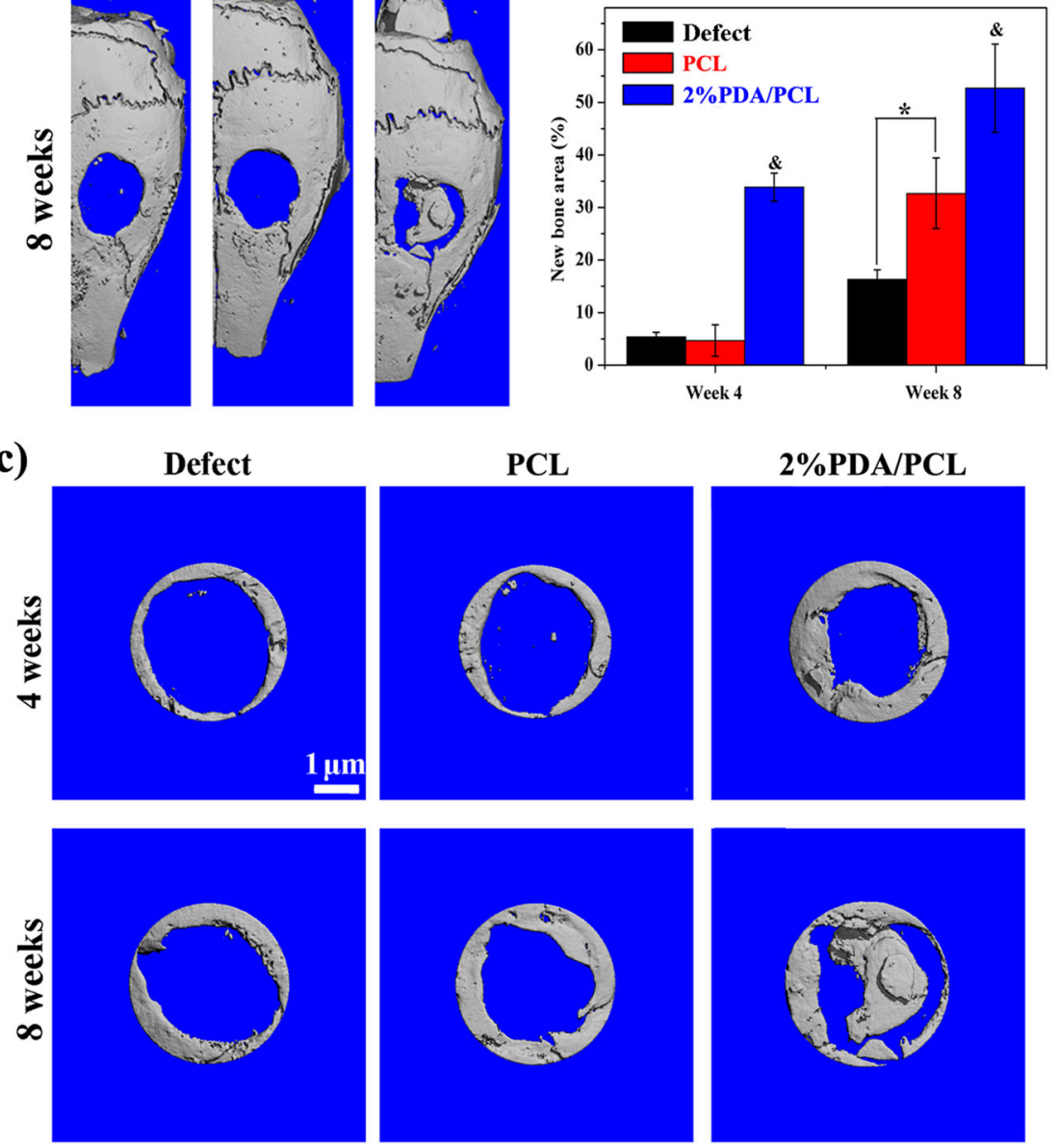

\section{2\%PDA/PCL}

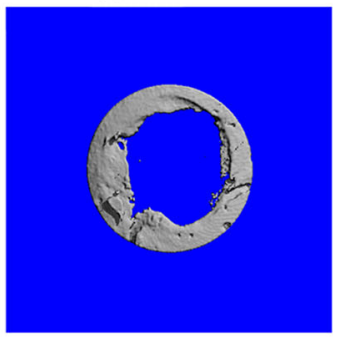

(d)
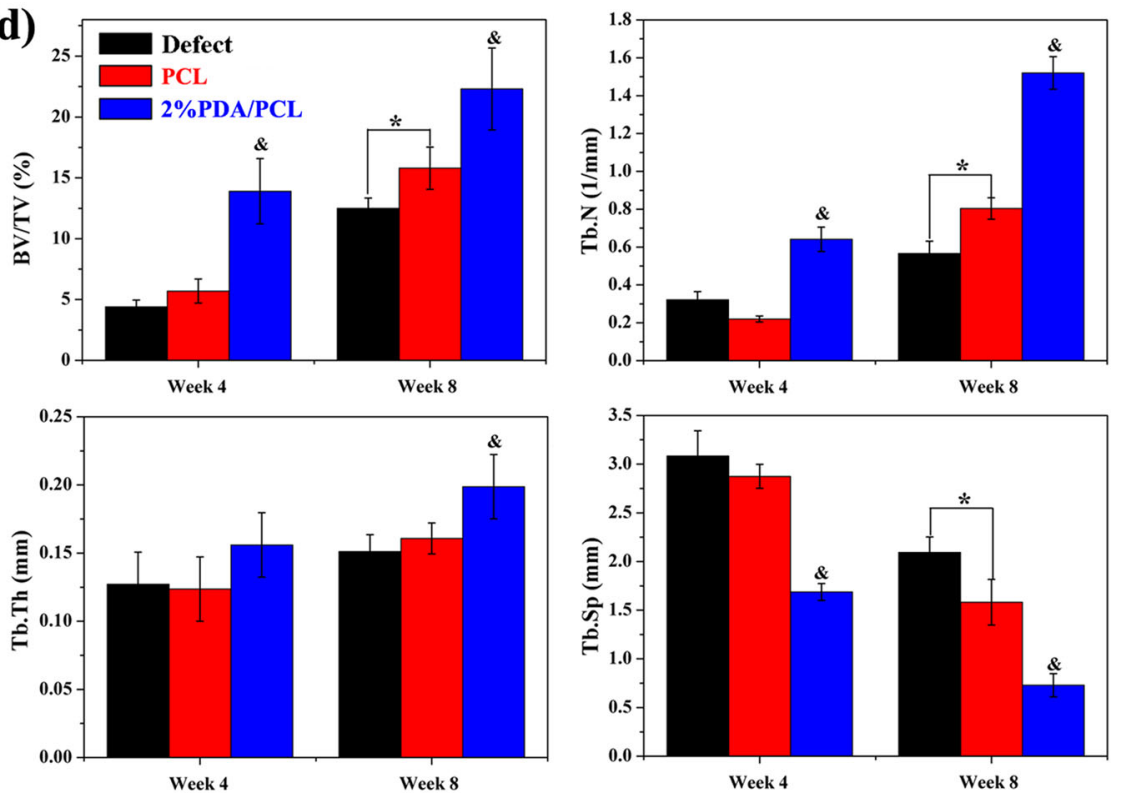

Fig. 7 In vivo evaluation of PDA/PCL fibrous membranes. a Reconstructed 3D micro-CT images of whole skulls after surgery at 8 weeks. $\mathbf{b}$ Quantification of new bone areas at 4 and 8 weeks. c Micro-CT images of the defects and $\mathbf{d}$ bone histomorphometry of membrane implants analyzed from the micro-CT data after 4 and 8 weeks. * represents $p<0.05$ between groups, and \& represents $p<0.05$ compared with other groups 


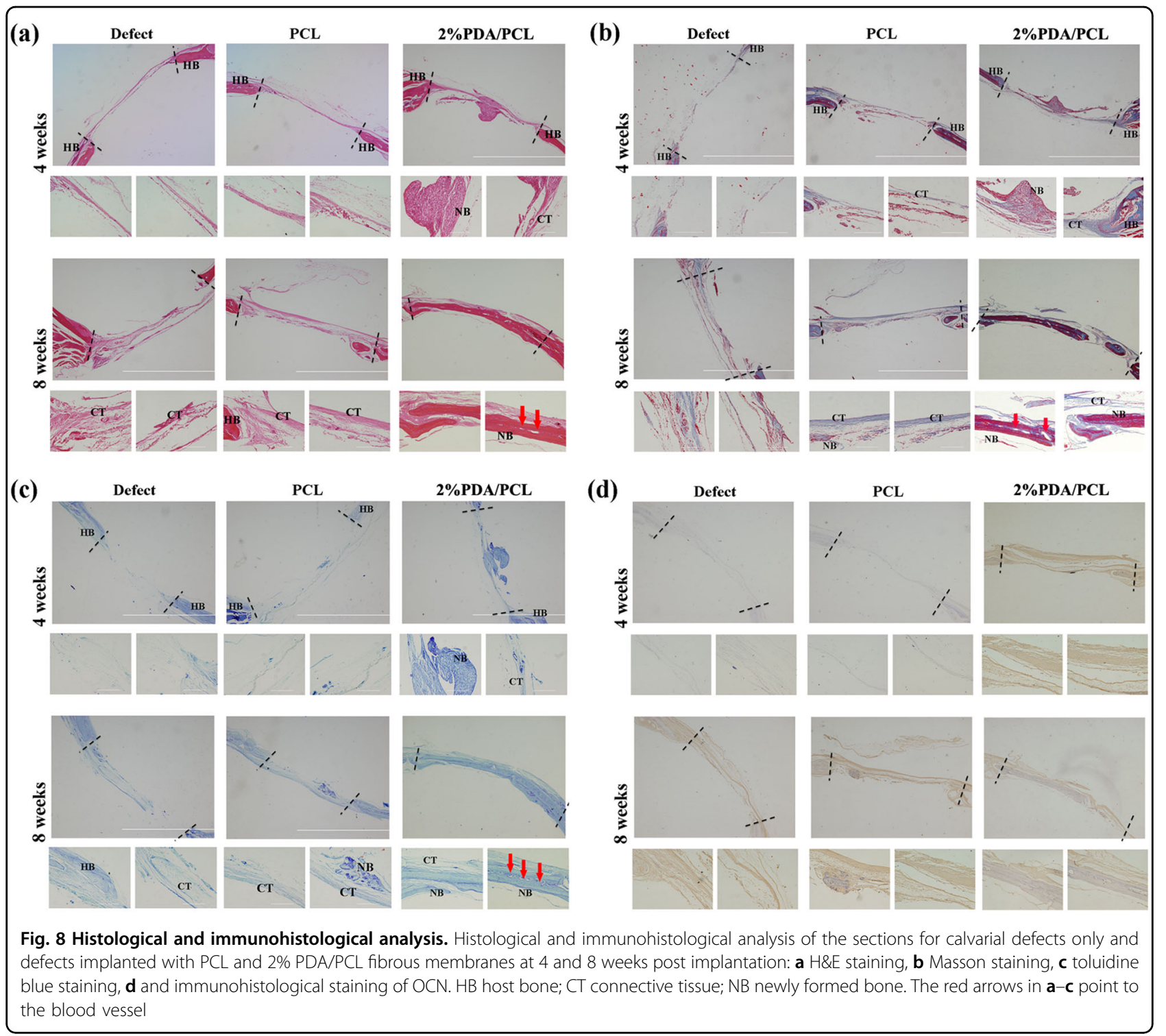

( $33.9 \pm 2.68 \%$ for 4 weeks and $52.7 \pm 8.36 \%$ for 8 weeks) and considerable lengths of new bone gradually expand to the center of the bone defect for the $2 \%$ PDA/PCL group.

Figure $7 \mathrm{~d}$ reveals the corresponding bone histomorphometry indices from the $3 \mathrm{D}$ micro-CT data. $\mathrm{BT} / \mathrm{TV}$, $\mathrm{Tb} . \mathrm{N}$, and $\mathrm{Tb}$. Th increased with decreased $\mathrm{Tb} . \mathrm{Sp}$ and were maintained in the $2 \%$ PDA/PCL group at both 4 and 8 weeks after surgery, demonstrating that the amount of new bone in defects covered by the 2\% PDA/PCL membranes was dramatically higher than that in the pure PCL counterparts. The fluorochrome staining assays further support the micro-CT results. More calcified matrix and bone remodeling are observed in the $2 \%$ PDA/PCL fibrous membranes (Fig. S6), which indicates phenomenal osteoinductive integration. Hence, the sequential fluorescent labeling suggests that the 2\% PDA decoration induces newly formed bone.

We further applied histological staining to identify the regenerated bone tissue in defects. H\&E staining revealed that at 4 and 8 weeks postoperation, fibrous tissue is the primary component in the cavities of the pure PCL group (Fig. 8a). In contrast, a visible bone structure is observed in the middle of cavities implanted with $2 \%$ PDA/PCL fibrous membranes. Moreover, bone with abundant vascularization was found after 8 weeks postoperation for the $2 \%$ PDA/PCL groups. Despite the fact that we do not obtain satisfying results from the H\&E staining results, some valuable information can be obtained from Masson (Fig. 8b), toluidine blue (Fig. 8c) and IHC staining (Fig. $8 d$ ) results. Masson staining shows that the defect-only 
group and PCL group have loosely formed connected tissue, and a portion of the mineralized collagen is enhanced in the PCL group (Fig. 8b and Fig. S7a). With regard to the $2 \%$ PDA/PCL fibrous membranes, new immature bone and more collagen formation are observed, which infiltrate the bone cavity at 4 weeks. At 8 weeks, implantation of $2 \%$ PDA/PCL fibrous membranes markedly enhances collagen formation, and newly formed bone nearly fully covers the defect area. In fact, only a small osteoid is found at the edge of defectimplanted pure PCL fibrous membranes according to the toluidine blue staining result, in which immature and mature bone structures can be stained blue. However, $2 \%$ PDA/PCL induces the regeneration of a mass of trabecular bones. IHC analysis also reveals that the highest production of $\mathrm{OCN}$ is expressed in the $2 \% \mathrm{PDA} / \mathrm{PCL}$ fibrous membranes (Fig. 8d and Fig. S7b). These histological results all demonstrate that the employment of PDA NPs in engineered fibrous membranes significantly boosts bone regeneration, which supports the hypothesis that the enhancement of in vitro osteo-differentiation is also effective in vivo.

\section{Conclusion}

In summary, by co-electrospinning PDA NPs with a bioinert synthetic polymer, we constructed bioinspired, flexible, and osteopromotive PDA/PCL fibrous membranes for bone regenerative medicine. The incorporation and amount of PDA NPs significantly influence the chemical composition, fiber size and mechanical properties of fibrous membranes. The cell experiment results indicate that the PDA/PCL fibrous membranes can facilitate the spreading, reproduction, and in vitro osteogenic commitment of hMSCs in a dose-dependent manner. In addition, in vivo preclinical evaluation validates that the quantity of newly formed bone with $2 \%$ PDA/PCL fibrous membranes is higher than that with pure PCL. Considering these data together, we demonstrate that the engineered PDA/PCL fibrous membranes, which are osteoinductive and easy to transplant, have great potential for GTR application.

\section{Acknowledgements}

This work is jointly funded by the State Key Laboratory of Military Stomatology (No. 2016KA01), National Natural Science Foundation of China (81801848), Sichuan Science and Technology Program (2017FZ0046, 2018JZ0026, and 2019YJ0554), China Postdoctoral Science Foundation (2017M610600), Chengdu International Science and Technology Cooperation Foundation (2017-GH02-00025-HZ), Hong Kong Scholar Programme, and Australian Research Council.

\section{Author details}

${ }^{1}$ Central Laboratory, Peking University School and Hospital of Stomatology, National Engineering Laboratory for Digital and Material Technology of Stomatology, Beijing 100081, China. ${ }^{2}$ School of Chemical Engineering and College of Materials Science and Engineering, Sichuan University, Chengdu 610065, China. ${ }^{3}$ Department of Mechanical Engineering, The University of
Hong Kong, Hong Kong SAR, China. ${ }^{4}$ Centre for Future Materials, University of Southern Queensland, Springfield Central, QLD 4300, Australia. ${ }^{5}$ Materials Engineering, The University of Queensland, Brisbane, QLD 4072, Australia. ${ }^{6}$ Laboratory for Biomaterials and Regenerative Medicine, Academy for Advanced Interdisciplinary Studies, Peking University, Beijing 100871, China

Conflict of interest

The authors declare that they have no conflict of interest.

\section{Publisher's note}

Springer Nature remains neutral with regard to jurisdictional claims in published maps and institutional affiliations.

Supplementary Information is available for this paper at https://doi.org/ 10.1038/s41427-019-0139-5.

Received: 13 March 2019 Revised: 24 April 2019 Accepted: 1 May 2019. Published online: 12 July 2019

\section{References}

1. Deng, Y. et al. A novel hydrogel surface grafted with dual functional peptides for sustaining long-term self-renewal of human induced pluripotent stem cells and manipulating their osteoblastic maturation. Adv. Funct. Mater. 28, 1705546 (2018).

2. Seftel, A. Treatment of infections associated with surgical implants. New Engl. J. Med. 172, 1422-1429 (2004).

3. Liu, J. et al. Pre-vascularization in fibrin Gel/PLGA microsphere scaffolds designed for bone regeneration. NPG Asia Mater. 10, 827-839 (2018).

4. Nasajpour, A. et al. A Multifunctional polymeric periodontal membrane with osteogenic and antibacterial characteristics. Adv. Funct. Mater. 28, 1703437 (2018).

5. Lee, Y. J. et al. Electrospun fibers immobilized with bone forming peptide-1 derived from BMP7 for guided bone regeneration. Biomaterials 34, 5059-5069 (2013).

6. Rim, N. G. et al. Modulation of osteogenic differentiation of human mesenchymal stem cells by poly[(L-lactide)-co-(epsilon-caprolactone)]/gelatin nanofibers. Macromol. Biosci. 9, 795-804 (2010).

7. Yong, B. K. \& Kim, G. H. Rapid-prototyped collagen scaffolds reinforced with PCL/ $\beta$-TCP nanofibres to obtain high cell seeding efficiency and enhanced mechanical properties for bone tissue regeneration. J. Mater. Chem. 22 16880-16889 (2012)

8. Ravichandran, R., Venugopal, J. R., Sundarrajan, S., Mukherjee, S. \& Ramakrishna, S. Precipitation of nanohydroxyapatite on PLLA/PBLG/Collagen nanofibrous structures for the differentiation of adipose derived stem cells to osteogenic lineage. Biomaterials 33, 846-855 (2012).

9. Ehsan, S., Masoud, S., Nasser, G. \& Iman, S. Nanohydroxyapatite-coated electrospun poly(l-lactide) nanofibers enhance osteogenic differentiation of stem cells and induce ectopic bone formation. Biomacromolecules 11, 3118-3125 (2010).

10. Chen, J. P. \& Chang, Y. S. Preparation and characterization of composite nanofibers of polycaprolactone and nanohydroxyapatite for osteogenic differentiation of mesenchymal stem cells. Colloids Surf. B 86, 169-175 (2011).

11. Deng, Y., Yang, Y. \& Wei, S. Peptide-decorated nanofibrous niche augments in vitro directed osteogenic conversion of human pluripotent stem cells. Biomacromolecules 18, 587-598 (2017).

12. $D, H ., M B, H . \& H, S$. A review of material properties of biodegradable and bioresorbable polymers and devices for GTR and GBR applications. Int. J. Oral. Max. Impl. 11, 667 (1996).

13. Xue, J. et al. Electrospun microfiber membranes embedded with drug-loaded clay nanotubes for sustained antimicrobial protection. ACS Nano 9, 1600-1612 (2015).

14. Teng, C. P. et al. Star-shaped polyhedral oligomeric silsesquioxanepolycaprolactone-polyurethane as biomaterials for tissue engineering application. NPG Asia Mater. 6, e142 (2014).

15. Haeshin, L., Dellatore, S. M., Miller, W. M. \& Messersmith, P. B. Mussel-inspired surface chemistry for multifunctional coatings. Science 318, 426-430 (2007). 
16. Kisuk, $Y$. et al. Polydopamine-mediated surface modification of scaffold materials for human neural stem cell engineering. Biomaterials 33, 6952-6964 (2012).

17. Ge, L. et al. Polydopamine-coated paper-stack nanofibrous membranes enhancing adipose stem cells' adhesion and osteogenic differentiation. J. Mater. Chem. B 2, 6917-6923 (2014).

18. Lin, C. C. \& Fu, S. J. Osteogenesis of human adipose-derived stem cells on poly (dopamine)-coated electrospun poly(lactic acid) fiber mats. Mater. Sci. Eng. C 58, 254 (2016).

19. Han, L. et al. Tough, self-healable and tissue-adhesive hydrogel with tunable multifunctionality. NPG Asia Mater. 9, e372 (2017).

20. Ping, Z. et al. Simple and versatile synthetic polydopamine-based surface supports reprogramming of human somatic cells and long-term self-renewa of human pluripotent stem cells under defined conditions. Biomaterials $\mathbf{8 7}, 1$ (2016).

21. Eap, S. et al. Polydopamine particles effect on melanoma cells proliferation and melanin secretion. Adv. Chem. Eng. Sci. 3, 1-10 (2013).

22. Hong, S. et al. Attenuation of the in vivo toxicity of biomaterials by polydopamine surface modification. Nanomedicine 6, 793-801 (2011).

23. Gao, X. et al. Bioinspired design of polycaprolactone composite nanofibers as artificial bone extracellular matrix for bone regeneration application. ACS Appl. Mater. Interfaces 8, 27594-276108 (2016).

24. Perikamana, S. K. M. et al. Effects of immobilized BMP-2 and nanofiber morphology on in vitro osteogenic differentiation of hMSCs and in vivo collagen assembly of regenerated bone. ACS Appl. Mater. Interfaces 7, 8798 (2015).
25. Rim, N. G. et al. Mussel-inspired surface modification of poly(l-lactide) electrospun fibers for modulation of osteogenic differentiation of human mesenchymal stem cells. Colloids Surf. B 91, 189-197 (2012).

26. Jia, Z. et al. Bioinspired anchoring AgNPs onto micro-nanoporous TiO2 orthopedic coatings: Trap-killing of bacteria, surface-regulated osteoblast functions and host responses. Biomaterials 75, $203-222$ (2016).

27. Lee, J. S., Yi, J. K., An, S. Y. \& Heo, J. S. Increased osteogenic differentiation of periodontal ligament stem cells on polydopamine film occurs via activation of integrin and PI3K signaling pathways. Cell. Physiol. Biochem. 34, 1824-1834 (2014).

28. Wei, D. et al. Soybean lecithin-mediated nanoporous PLGA microspheres with highly entrapped and controlled released BMP-2 as a stem cell platform. Small 14, 1800063 (2018).

29. $\mathrm{Wu}, \mathrm{H}$. et al. Genomic occupancy of Runx2 with global expression profiling identifies a novel dimension to control of osteoblastogenesis. Genome Biol. 15, R52 (2014).

30. Xu, A. et al. A carboxymethyl chitosan and peptide-decorated polyetheretherketone ternary biocomposite with enhanced antibacterial activity and osseointegration as orthopedic/dental implants. J. Mater. Chem. B 4, 1878 (2016).

31. Kaneto, C. M. et al. COL1A1 and miR-29b show lower expression levels during osteoblast differentiation of bone marrow stromal cells from osteogenesis Imperfecta patients. BMC Med. Genet. 15, 45 (2014).

32. $\mathrm{Ma}, \mathrm{H}$. et al. $3 \mathrm{D}$ printing of high-strength bioscaffolds for the synergistic treatment of bone cancer. NPG Asia Mater. 10, 31-44 (2018). 